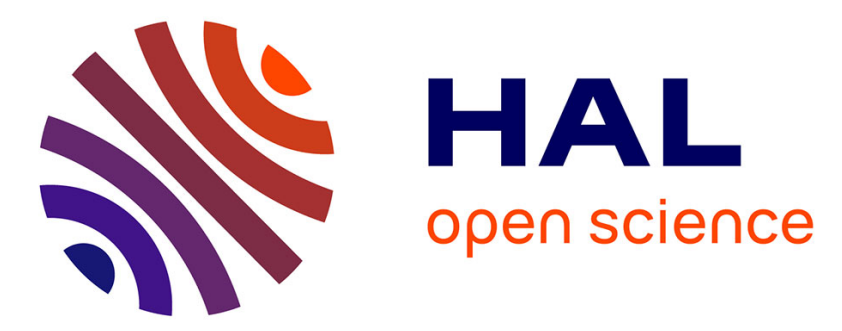

\title{
Graph-based flow modeling approach adapted to multiscale discrete-fracture-network models
}

Diane Doolaeghe, Philippe Davy, Jeffrey D Hyman, Caroline Darcel

\section{To cite this version:}

Diane Doolaeghe, Philippe Davy, Jeffrey D Hyman, Caroline Darcel. Graph-based flow modeling approach adapted to multiscale discrete-fracture-network models. Physical Review E , 2020, 102 (5), pp.053312. 10.1103/PhysRevE.102.053312 . insu-03020746

\section{HAL Id: insu-03020746 \\ https://hal-insu.archives-ouvertes.fr/insu-03020746}

Submitted on 24 Nov 2020

HAL is a multi-disciplinary open access archive for the deposit and dissemination of scientific research documents, whether they are published or not. The documents may come from teaching and research institutions in France or abroad, or from public or private research centers.
L'archive ouverte pluridisciplinaire HAL, est destinée au dépôt et à la diffusion de documents scientifiques de niveau recherche, publiés ou non, émanant des établissements d'enseignement et de recherche français ou étrangers, des laboratoires publics ou privés. 


\title{
Graph-based flow modeling approach adapted to multiscale discrete-fracture-network models
}

\author{
Diane Doolaeghe $\odot,{ }^{1,2, *}$ Philippe Davy $\odot,{ }^{1, \dagger}$ Jeffrey D. Hyman $\odot,{ }^{3, \ddagger}$ and Caroline Darcel ${ }^{2, \S}$ \\ ${ }^{1}$ Géosciences Rennes, OSUR, CNRS, Université de Rennes 1, 263 Avenue Général Leclerc, 35042 Rennes, France \\ ${ }^{2}$ Itasca Consultants S.A.S., 64 Chemin des Mouilles, 69130 Ecully, France \\ ${ }^{3}$ Computational Earth Science Group (EES-16), Earth and Enviromental Sciences Division, Los Alamos National Laboratory, P.O. Box 1663, \\ Los Alamos, New Mexico 87545, USA
}

(Received 7 July 2020; accepted 30 October 2020; published 20 November 2020)

\begin{abstract}
Fractured rocks are often modeled as multiscale populations of interconnected discrete fractures (discrete fracture network, DFN). Graph representations of DFNs reduce their complexity to their connectivity structure by forming an assembly of nodes connected by links (edges) to which physical properties, like a conductance, can be assigned. In this study, we address the issue of using graphs to predict flow as a fast and relevant substitute to classical DFNs. We consider two types of graphs, whether the nodes represent the fractures (fracture graph) or the intersections between fractures (intersection graph). We introduce an edge conductance expression that accounts for both the portion of the fracture surface that carries flow and fracture transmissivity. We find that including the fracture size in the expression improves the prediction of flow compared to expressions used in previous studies that did not. The two graph types yield very different results. The fracture graph systematically underestimates local flow values. In contrast, the intersection graph overestimates the flow in each fracture because of the connectivity redundancy in fractures with multiple intersections. We address the latter issue by introducing a correction factor into the conductances based on the number of intersections on each fracture. We test the robustness of the proposed conductance model by comparing flow properties of the graph with high-fidelity DFN simulations over a wide range of network types. The good agreement found between the intersection graph and test suite indicates that this representation could be useful for predictive purposes.
\end{abstract}

DOI: 10.1103/PhysRevE.102.053312

\section{INTRODUCTION}

Graphical representations of complex systems have seen rapidly growing interest over the last few decades [1]. Graphs are mathematical structures where a set of discrete entities are represented as nodes connected by a set of edges based on a particular rule. This compact mathematical structure enables the detailed study of the connectivity of an ensemble of connected discrete objects and has been applied to a wide range of fields including, for example, the World Wide Web [2], scientific collaboration networks [3], and biologic systems [4].

Recently, graphs have gained attention in the scientific field of subsurface fracture network modeling [5-7]. In environments where the matrix permeability is negligible, like in crystalline rocks, fracture networks define permeability and flow path organization. These fracture networks are complex and are composed of intersecting discrete fractures, that are characterized by a wide distribution of sizes and apertures. More specifically for crystalline rocks, fracture sizes likely follow a power-law distribution [8]. The resulting multiscale structure makes it difficult to define a characteristic length scale and perform a classic hydraulic characterization with continuum approaches [9-11]. In contrast to more homoge-

\footnotetext{
${ }^{*}$ Corresponding author: d.doolaeghe@itasca.fr

Corresponding author: philippe.davy@univ-rennes1.fr

‡Corresponding author: jhyman@lanl.gov

${ }^{\S}$ Corresponding author: c.darcel@itasca.fr
}

neous subsurface systems, details of the network structures are a principal control of the flow of fluids therein $[12,13]$. The critical role played by the network structure on the flow stimulates, therefore, the use graphs for investigating the fracture network's hydraulic properties. Graphs provide a compact framework for improved characterization of the structure of fracture networks, which is critical for numerous applications including the disposal of spent nuclear fuel [14], hydrogeology [15], and geothermal [16] and petroleum resources [17,18].

The use of graphs is all the more necessary as the number of fractures required to describe a geological environment is huge. Due to the multiscale nature of fracture networks, it may be necessary to model billions of fractures to understand which fractures are significant. For instance, a realistic fracture network with conditions described in Davy et al. [19] (power-law size distribution exponent of -4 , dimensionless density of 3) has $10^{9}$ fractures with sizes ranging from $1 \mathrm{~m}$ (a size that cannot be considered negligible) to $1 \mathrm{~km}$ (a typical size of domains for most of groundwater applications). This casual approximation gives an idea of the complexity of the networks that need to be managed. The discretization of the full fracture network, as done in discrete fracture network (DFN) models (see Lei et al. [20] for a review) poses computational problems. This is not the only reason why graphs are interesting intermediate steps in the modeling of fractured media, but it is already a strong enough reason.

Several studies have investigated the possibility of using a graph to decrease the computational burden associated with 
DFN flow and transport simulations. In the past, when technological progress did not afford the numerical resources necessary for DFN modeling, pipe networks, analogous to graphs, were used as an alternative basis [21-24]. A major issue was to render the pipe network, composed of one-dimensional pipes hydraulically equivalent to a DFN. Therefore, mixed analytical-numerical techniques have been developed, such as using the boundary element method to deduce pipe conductances [23], or using the Image theory to account for impermeable fracture borders on the flow [25]. More recently, graphs have been used as a complement to DFN flow and transport simulations [26]. They have been successfully used to identify the DFN backbone, i.e., fractures participating to shortest travel times or carrying the major flows [27-31]. They have also been used to directly solve flow and transport [32] in order to reduce the cost associated with uncertainty quantifications (e.g., multifidelity Monte Carlo, O’Malley et al. [33], and multilevel Monte Carlo, Berrone et al. [34]). In these recent studies, simple analytical definitions were given as edges hydraulic attributes to preserve the rapidity (quasi-immediate) of the graph computations. A key element in creating a graph that provides predictions of flow and transport similar to the higher fidelity DFN is assigning an edge conductance that is equivalent to the fracture surface in the DFN. Previous definitions have included the physical distance between nodes [27] and the intersection sizes [32] to simplify the flowing regions between fractures. These geometric simplifications resulted in marked differences between the DFN and graph flow models. To date, few efforts have been made to include higher-order geometric information in the graph edge conductance properties.

In the present study, we address the issue of defining an equivalent conductance for graph edges in the case of diskshaped fractures with a constant transmissivity by fracture. A DFN can be transformed into two graph types: a fracture graph [21,22,28], in which vertices represent fractures and edges connect two intersecting fractures, and an intersection graph [23,32], in which nodes represent intersections and edges connect two intersections of the same fracture. Another possible type is the bipartite graph, in which two disjoint sets of nodes, one representing fractures and the other intersections, are connected and the former two graph types can be readily obtained [27]. In this study, we focus on the first two graph representations. We provide analytical formulations of the equivalent conductance between two fractures (for the fracture graph edges) and between two intersections (for the intersection graph edges). The conductance $\left(\mathrm{m}^{2} \mathrm{~s}^{-1}\right)$ measures how effectively the fluid, subjected to a potential difference, is transported through a medium. It is related to the flow, $Q$ $\left(\mathrm{m}^{3} \mathrm{~s}^{-1}\right)$, and the hydraulic potential (head) difference, $\Delta H$ (m), with the formulation $Q=C \Delta H$. By observing that the fracture flow field is often limited by the fracture impervious borders, we propose formulations that include fracture sizes in addition to the physical distances, intersections sizes, and fracture apertures. Considering the wide range of fracture sizes in nature, this definition can make large differences compared to previously presented ones.

Our conductance is verified against classical DFN flow simulation via comparison with flow on entirely meshed fracture surfaces. It is first verified locally using a single fracture with two intersections but varying geometries. Next, we consider DFNs composed of hundreds to tens of thousands of fractures. We transform large DFNs into equivalent fracture graphs and intersection graphs and resolve the flow on the graphs and the DFNs. The graph estimations are compared to the classic DFN flow solving at two scales: at the DFN scale, by comparing equivalent permeabilities, and at the fracture scale, by comparing the total flow exchanged by fractures [35]. The verification at the fracture scale allows us to evaluate if the internal network flowing structure is being properly represented by the graphs. As the conductance is originally defined for ideal cases of fractures having two intersections, there are biases in the graph due in part to the DFN connectivity, i.e., fractures having more than two intersections and participating in many structural paths. Therefore, we test the method on several DFN types with increasing connectivity complexity: constant fracture size DFN, power-law size distributed DFN, and genetic networks with small intersections (UFM, universal fracture model) [19,36].

The remainder of the paper is organized as follows. Section II describes the technical aspects of the study: graph constructions, flow solving, and measurement of the flow metrics. Section III presents the developments of the equivalent conductances for both graph types. Section IV presents and analyses the results for the different DFN types mentioned above. Finally, conclusions are drawn in Sec. V.

\section{METHODOLOGY}

DFN models are explicit representations of fracture networks and can be used as a support for physical simulations, including flow and transport. The way the fractures, represented by finite planar objects, intersect in the threedimensional volume impacts the hydrological properties of the network $[13,35]$. A DFN can be either stochastically generated with probability distribution derived from the field (densities, size distribution, set of orientations [37]) or with models mimicking the geological fracturing process (UFM $[19,36])$. In this study, we use the computational suite DFN.lab [38] to generate DFNs in cubic systems. We generate DFNs with varying properties, like the fracture size distribution or the connectivity quantified by the percolation parameter. More details on the DFNs generated for this study are given later in Sec. IV.

DFNs lend themselves to the construction of graphs. Graphs are representations consisting of nodes connected by edges. One edge connects two nodes if an exchange physically exists between these nodes. The exchange intensity can be modulated by assigning attributes to the edges, such as a conductance. We present in the next section the details of the construction of two graph types from a DFN, a fracture graph and an intersection graph, and the notations that we use to qualify nodes, edges, and their attributes.

\section{A. Graph constructions \\ 1. Fracture graph}

Let $F=\left\{f_{i}\right\}$ for $i=1, \ldots, n$ denote a DFN composed of $n$ fractures. We define a mapping $\varphi$, that transforms $F$ into a graph $G(U, E)$. For every $f_{i} \in F$, there is a unique vertex 
(a)

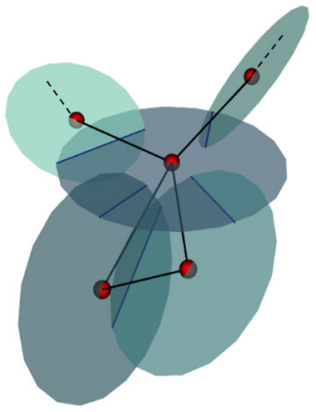

(b)

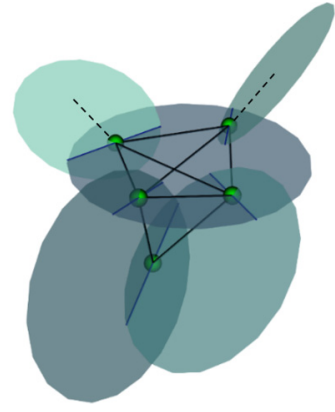

FIG. 1. DFNs and equivalent graphs: (a) fracture graph, (b) intersection graph.

$u_{i} \in U$

$$
\varphi: F \rightarrow u_{i}
$$

If two fractures, $f_{i}$ and $f_{j}$, intersect $\left(f_{i} \cap f_{j} \neq \emptyset\right)$, then there is an edge in $E$ connecting the corresponding vertices,

$$
\varphi: I=\left(f_{i} \cap f_{j}\right) \neq \emptyset \rightarrow e_{i j}=\left(u_{i}, u_{j}\right) .
$$

For every intersection between a fracture $f_{i}$ and a plane boundary $L$, there is a unique vertex $u_{i L} \in V$,

$$
\varphi:\left(f_{i} \cap L\right) \neq \emptyset \rightarrow u_{i L}
$$

and a unique edge $e_{i L}$,

$$
\varphi:\left(f_{i} \cap L\right) \neq \varnothing \rightarrow e_{i L}=\left(u_{i}, u_{i L}\right) .
$$

For every $\left(f_{i}, f_{j}\right) \in F$ and $f_{i} \cap f_{j} \neq \emptyset$, we attribute to each edge $e_{i j} \in E$ an attribute called conductance $C_{i j}$. A definition of $C_{i j}$ is presented later in Sec. III. An image of the graph mapping $\varphi$ is presented in Fig. 1(a).

\section{Intersection graph}

We define a second mapping $\psi$, that transforms $F$ into a graph $G(V, E)$. For every $\left(f_{i}, f_{j}\right) \in F$, there is a unique vertex $v_{i j} \in V$,

$$
\psi: I=\left(f_{i} \cap f_{j}\right) \neq \emptyset \rightarrow v_{i j} .
$$

If a fracture $f_{i}$ intersects two fractures $f_{j}$ and $f_{k}\left(f_{i} \cap f_{j} \neq\right.$ $\emptyset$ and $\left.f_{i} \cap f_{k} \neq \emptyset\right)$, then there is an edge in $E$ connecting the corresponding vertices,

$$
\psi:\left(\left(f_{i} \cap f_{j}\right) \neq \emptyset \text { and }\left(f_{i} \cap f_{k}\right) \neq \emptyset\right) \rightarrow e_{j k}^{i}=\left(v_{i j}, v_{i k}\right) .
$$

For every intersection between a fracture $f_{i}$ and a boundary $L$, there is a unique vertex $v_{i L} \in V$,

$$
\psi:\left(f_{i} \cap L\right) \neq \emptyset \rightarrow v_{i L}
$$

and a unique edge $e_{j L}^{i}$,

$$
\left.\psi:\left(\left(f_{i} \cap L\right) \neq \emptyset \quad \text { and } \quad\left(f_{i} \cap f_{j}\right) \neq \emptyset\right)\right) \rightarrow e_{j L}^{i}=\left(v_{i j}, v_{i L}\right) \text {. }
$$

We attribute to each edge $e_{j k}^{i} \in E$ a conductance $C_{j k}^{i}$. A definition of $C_{j k}^{i}$ is presented later in Sec. III. An image of the graph mapping $\psi$ is presented in Fig. 1(b).

\section{B. Flow and metrics}

In this section, we present how we solve flow within the DFNs and graphs. Once the steady-state flow fields are obtained, we compute the following flow indicators: the bulk permeability $K\left(\mathrm{~m} \mathrm{~s}^{-1}\right)$ and the total flow exchanged by fracture $Q_{f}\left(\mathrm{~m}^{3} \mathrm{~s}^{-1}\right)$ for comparison [35].

\section{Flow solution}

Steady-state flow is simulated with permeameter-like boundary conditions, i.e., two opposite faces of the system are assigned fixed head boundary conditions, and the remaining face a zero flow condition. For the reference computation, the DFNs are entirely meshed and the flow is solved using a mixed hybrid finite element method. We refer to Pinier et al. [38] for details about mesh generation and flow solving. The equivalent graph flow is solved by using the methodology described in Karra et al. [32]. Below we present an outline of the method and refer to Karra et al. [32] for more information. Boundary conditions are first provided, i.e., constant heads are attributed to the boundary vertices, and vertices corresponding to a no-flow boundary are deleted from the graph. Heads (m) are solved in the vertex set by solving the flow conservation at each vertex. Finally, edge flows $\left(\mathrm{m}^{3} \mathrm{~s}^{-1}\right)$ are deduced from the edge adjacent vertex heads and the edge conductances.

In the remainder of the paper, we use the terminologies "complete simulation" and "graph simulation" to qualify the flow solved with respectively the complete meshed DFN and the equivalent graphs.

\section{Flow metrics}

In this study, we compare flow between the graph simulations and the complete simulations at two scales. At the larger of the two, the DFN scale, we compute the bulk permeability $K\left(\mathrm{~m} \mathrm{~s}^{-1}\right)$. At the smaller of the two, that of the fracture scale, we compute the total flow exchanged by fracture, $Q_{f}\left(\mathrm{~m}^{3} \mathrm{~s}^{-1}\right)$ :

$$
Q_{f}=\frac{1}{2} \sum_{j} Q_{i j},
$$

with $Q_{i j}\left(\mathrm{~m}^{3} \mathrm{~s}^{-1}\right)$ the flow exchanged through the intersection between $f_{i}$ and $f_{j}$. These metrics are provided by the DFN.lab suite [38].

In the graph simulations, the bulk permeability $K$ is deduced from the network total flow, $Q_{T}$, and system dimensions. In the following, we give details on how we compute the fracture flow $Q_{f}$ in the graphs, because the method is different in each graph type. In the fracture graph, the $Q_{f}$ are deduced from the edge flows, $Q\left(e_{i j}\right)$, directly equal to the intersection flows,

$$
\varphi: Q_{i j}=Q\left(e_{i j}\right)
$$

In the intersection graph, the intersection flow $Q_{i j}$ is obtained differently because in this graph type intersections are represented by a vertex. Besides, a share of the total flow that moves through the vertex may remain on the same fracture, so, physically, it does not cross the intersection represented by this vertex (an example is illustrated in Fig. 2). To compute $Q_{i j}$, we select the edges on one side of the intersection belonging either to fracture $f_{i}$ or to fracture $f_{j}$. Then, we add all the 


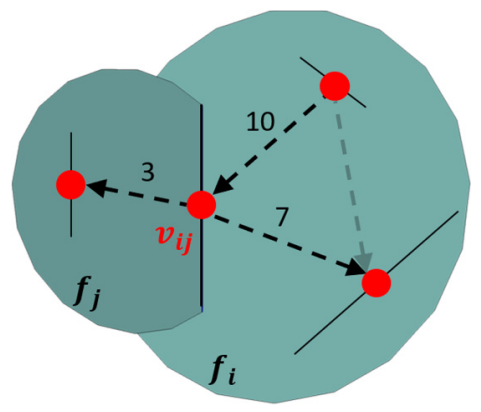

FIG. 2. Example of DFN equivalent intersection graph with the oriented flow edge attribute $Q\left(\mathrm{~m}^{3} \mathrm{~s}^{-1}\right)$ being indicated. The flow values in this example do not rely on any physical simulations but serve to illustrate how, in this graph type, a share of vertex total flows can remain on the same fracture. Here, from the edge with flow equal to $10 \mathrm{~m}^{3} \mathrm{~s}^{-1}$ only $3 \mathrm{~m}^{3} \mathrm{~s}^{-1}$ cross the intersection represented by the vertex $v_{i j}$ while $7 \mathrm{~m}^{3} \mathrm{~s}^{-1}$ remain on the same fracture $f_{i}$.

edge flows directed toward the vertex and we subtract the ones directed away from it. Finally, we take the absolute value of the result. On a fracture $f_{i}$, the flow $Q_{i j}$ through an intersection with fracture $j$ is computed in the intersection graph as

$$
\psi: Q_{i j}=\left|\sum_{k} \hat{Q}\left(e_{j k}^{i}\right)\right|
$$

$\hat{Q}\left(e_{j k}^{i}\right)$ is the edge $e_{j k}^{i}$ directed flow. The direction of the flow depends on the adjacent vertex heads, $H\left(v_{i j}\right)$ and $H\left(v_{i k}\right)$,

$$
\psi:\left\{\begin{array}{l}
\hat{Q}\left(e_{j k}^{i}\right)=+Q\left(e_{j k}^{i}\right) \text { if } H\left(v_{i j}\right)>H\left(v_{i k}\right), \\
\hat{Q}\left(e_{j k}^{i}\right)=-Q\left(e_{j k}^{i}\right) \text { if } H\left(v_{i j}\right)<H\left(v_{i k}\right),
\end{array}\right.
$$

where $Q\left(e_{j k}^{i}\right)$ is the edge $e_{j k}^{i}$ flow magnitude.

The equivalent permeability $K$ and the total flow by fracture $Q_{f}$ will be used later in Sec. IV to compare the graph simulations to the complete simulations.

\section{EDGE CONDUCTANCE}

In this section, we provide new analytical developments of the graph edge conductance with the aim of them being equivalent to the fracture flowing surface that the edge represents. By observing that the fracture flow field is often limited by the fracture impervious borders, we propose a definition of the conductance that includes the fracture size. This section is organized as follows: after presenting the schematical representations that support our developments (Sec. III A 1), we derive the conductance definition, first, for edges in the fracture graph (Sec. III A 2) and, second, for edges in the intersection graph (III A 3). Finally, we verify the analytical developments by comparing them to high-fidelity simulations of the flow on a fracture with two intersections (Sec. III B).

\section{A. Analytical definitions}

\section{Schematical representation of the fracture flowing surface}

In this study, fractures are modeled by disks. Thus, the diameter is the appropriate length to include in the modified definition of conductance. For both graph types, we
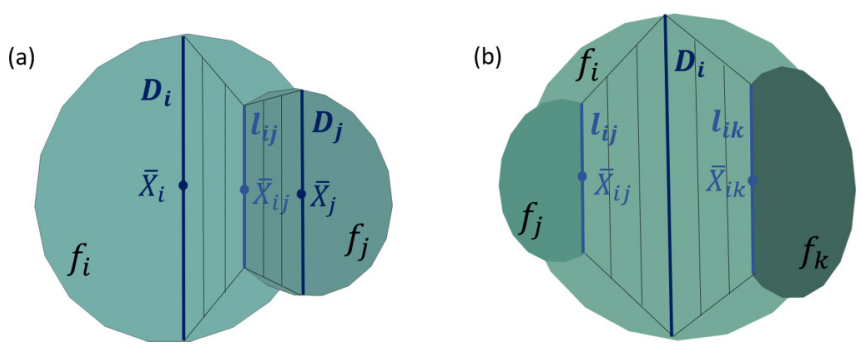

FIG. 3. Schematical representation of the fracture(s) flowing area(s) using trapezoïds. The notations used in the analytical developments of the conductance are also presented. (a) refers to the fracture graph and (b) to the intersection graph.

include the fracture diameter in the conductance definitions by schematically representing the flowing area as a series association of two trapezoids, presented in Fig. 3(a) for the fracture graph and Fig. 3(b) for the intersection graph. We derive an expression of the conductance $C\left(\mathrm{~m}^{2} \mathrm{~s}^{-1}\right)$ between the large base and the small base of one trapezoid to be

$$
C=T \frac{l_{B}-l_{b}}{x_{B b}\left[\ln \left(l_{B}\right)-\ln \left(l_{b}\right)\right]},
$$

where $l_{B}, l_{b}, x_{B b}(\mathrm{~m})$ are respectively the larger base size, the smaller base size, and the height of the trapezoid, and $T$ is the plane hydraulic transmissivity $\left(\mathrm{m}^{2} \mathrm{~s}^{-1}\right)$. The function $\ln (x)$ is the base $e$ logarithm. Additional details on how Eq. (13) is derived are presented in the Appendix.

Note that the schematic representations in Fig. 3 are ideal geometrical cases, where intersections are parallel. In reality, more complex geometries are found in DFNs, as intersections are often not parallel, possibly crossing, and their positions can be anywhere on the fracture plane. Later, in Sec. III B, we verify how the simplification proposed here works for intersections randomly placed on a fracture. However, this verification is limited to the case where the two intersections do not cross on the fracture plane because, in the numerical setup of Sec. III B, the two intersections have different boundary conditions and cannot share a common point. As the conductance is inversely proportional to the distance between intersections, the equivalent conductance in a configuration when intersection do cross should be underestimated by the conductance definition based on parallel and noncrossing intersections.

\section{Fracture graph}

The equivalent conductance $C$ of two conductances $C_{1}$ and $C_{2}$ in series association is $C=\left(\frac{1}{C_{1}}+\frac{1}{C_{2}}\right)^{-1}$. From Eq. (13), we derive an expression of the conductance $C_{i j}\left(\mathrm{~m}^{2} \mathrm{~s}^{-1}\right)$ attributed to the edge $e_{i j}$ in the fracture graph,

$$
\varphi: C_{i j}=\left(\frac{\left\|\bar{X}_{i}-\bar{X}_{i j}\right\| \ln \left(\frac{l_{i j}}{D_{i}}\right)}{T_{i}\left(l_{i j}-D_{i}\right)}+\frac{\left\|\bar{X}_{j}-\bar{X}_{i j}\right\| \ln \left(\frac{l_{i j}}{D_{j}}\right)}{T_{j}\left(l_{i j}-D_{j}\right)}\right)^{-1} .
$$

$\bar{X}_{i}, D_{i}$, and $T_{i}\left(\mathrm{~m}^{2} \mathrm{~s}^{-1}\right)$ are respectively the fracture $f_{i}$ center, size, and transmissivity. $\bar{X}_{i j}$ and $l_{i j}$ are respectively the intersection $\left(f_{i} \cap f_{j} \neq \emptyset\right)$ center and size [Fig. 3(a)]. 


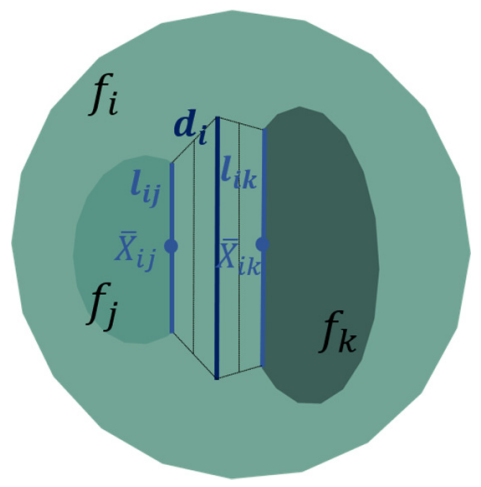

FIG. 4. Schematical representation of the flowing surface between two intersections in the case of close intersections.

For every intersection between a fracture $f_{i}$ and boundary $L$, if $f_{i} \cap L \neq \emptyset$, we attribute to each edge $e_{i L} \in E$ a conductance $C_{i L}$ :

$$
\varphi: C_{i L}=\left(\frac{\left\|\bar{X}_{i}-\bar{X}_{i L}\right\| \ln \left(\frac{l_{i L}}{D_{i}}\right)}{T_{i}\left(l_{i L}-D_{i}\right)}\right)^{-1}
$$

$\bar{X}_{i L}$ and $l_{i L}$ are respectively the intersection $\left(f_{i} \cap L \neq \emptyset\right)$ center and size.

\section{Intersection graph}

Based on the same reasoning as for the fracture graph, we derive an expression of the conductance $C_{j k}^{i}$ attributed to the edge $e_{j k}^{i}$ in the intersection graph,

$$
\psi: C_{j k}^{i}=\frac{2 . T_{i}}{\left\|\bar{X}_{i k}-\bar{X}_{i j}\right\|}\left(\frac{\ln \left(\frac{D_{i}}{l_{i j}}\right)}{\left(D_{i}-l_{i j}\right)}+\frac{\ln \left(\frac{D_{i}}{l_{i k}}\right)}{\left(D_{i}-l_{i k}\right)}\right)^{-1} .
$$

$D_{i}$ and $T_{i}\left(\mathrm{~m}^{2} \mathrm{~s}^{-1}\right)$ are respectively the fracture $f_{i}$ size, and transmissivity. $\bar{X}_{i j}$ and $l_{i j}$ are respectively the intersection $\left(f_{i} \cap f_{j} \neq \emptyset\right)$ center and size [Fig. 3(b)].

In this formulation, the position of the fracture center is not required. We choose to use the direct distance between the intersections, $\left\|\bar{X}_{i k}-\bar{X}_{i j}\right\|$, because it better represents the flowing distances.

Another adjustment is made possible in the intersection graph by the fact that the two intersections are directly connected. It regards the case where the two intersections are close enough so that the use of the fracture diameter $D_{i}$ overestimates the flowing surface and thus the conductance. In Fig. 4, we have schematically represented the flowing area between two close intersections. In this case, instead of $D_{i}$, a smaller size $d_{i}$ is more appropriate to schematize the flowing surface. Therefore, we replace $D_{i}$ by $\hat{D}_{i}$ in the formulation of $C_{j k}^{i}$ with

$$
\hat{D}_{i}=\min \left(D_{i}, d_{i}\right)
$$

$d_{i}$ is a function of intersection sizes and intersection distance,

$$
d_{i}=\frac{l_{i j}+l_{i k}}{2}+B\left\|\bar{X}_{i j}-\bar{X}_{i k}\right\| .
$$
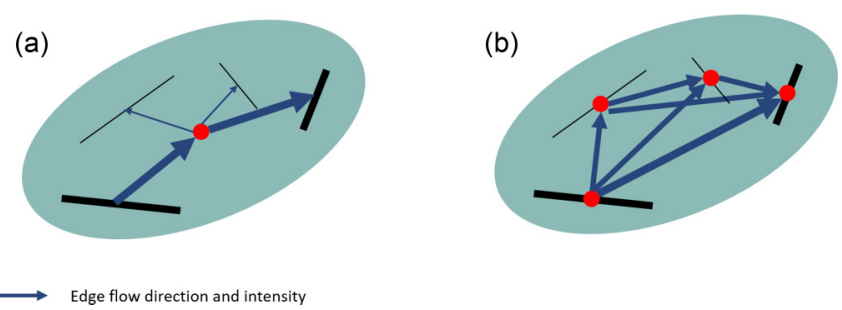

FIG. 5. Fracture with four intersections and equivalent fracture graph (a) and intersection graph (b). The two thick intersections are the most flowing on the fracture. The edge thicknesses and arrows represent the intensity and direction of the edge flows.

In Eq. (18) we adjust the coefficient $B$ by comparing our analytical definition to real numerical simulations of the flow between two intersections on a fracture, by varying intersection sizes and distance. It is found to be $\sim 1.5$.

\section{Multiple-paths correction in the intersection graph}

For fractures with more than two intersections, the intersection graph represents the fracture as an $n$-clique, with $n$ being equal to the number of intersections on the fractures. An $n$-clique is a subset of $n$ vertices such that every two vertices in the clique are adjacent. The problem is that this specific architecture creates a multiplicity of paths from one intersection vertex to another on the same fracture, thereby artificially increasing the equivalent volume through which the fluid flows. The effect of multiple paths is illustrated in Fig. 5 where a fracture with four intersections is represented with the fracture graph mapping [Fig. 5(a)] and the intersection graph mapping [Fig. 5(b)], along with edge flow intensities (line widths) and directions (arrows). In the example, there is one principle flow path formed by two intersections (thick intersections) among the four intersections. In the fracture graph (a), a unique path is possible between the two intersections. In the intersection graph (b), possible secondary paths are passing through other vertices in addition to the direct path between the two intersection vertices, which increases the amount of flow between the two intersections. To correct for this effect in the intersection graph, we reduce each edge conductance $C_{j k}^{i}$ of a fracture $f_{i}$ by a factor based on the number of intersections on that fracture, $n_{i}$. In the intersection graph, a vertice $v_{i j}\left(f_{i} \cap f_{j} \neq \emptyset\right)$ has a degree equal to $n_{i}-1$ on the fracture $f_{i}$,

$$
\psi: d\left(\left.v_{i j}\right|_{e_{j k}^{i} \in f_{i}}\right)=n_{i}-1
$$

Therefore, for each edge $e_{j k}^{i} \in f_{i}$, we reduce the conductance $C_{j k}^{i}$ as follows:

$$
\psi: \hat{C}_{j k}^{i}=\frac{C_{j k}^{i}}{n_{i}-1}
$$

Note that the correction only changes the conductances for fractures with more than two intersections. Later, in Sec. IV, we observe the efficiency of this correction for a large DFNs. 


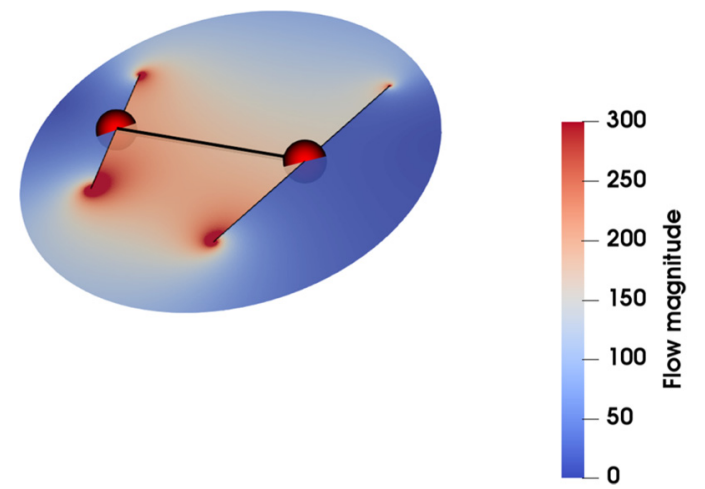

FIG. 6. Flow magnitude $\left(\mathrm{m}^{3} \mathrm{~s}^{-1}\right)$ numerically solved (software DFN.lab) in a single fracture having two intersections. The fracture diameter $(\mathrm{m})$ and transmissivity $\left(\mathrm{m}^{2} \mathrm{~s}^{-1}\right)$ are both equal to 1 . Constant heads $(\mathrm{m})$ are fixed on each intersection and the head difference is equal to 1 . An equivalent intersection graph is also represented (red vertices).

\section{B. Verification on a fracture with two intersections}

The conductance definitions developed in Sec. III A are verified using a single fracture with two intersections (Fig. 6). Steady-state flow is resolved on a computational mesh representation of that fracture, with head boundary conditions fixed on the two intersection segments (1 and 0$)$. With this setup, we only compute the equivalent conductance between two intersections and not between two fractures. However, as the two conductance definitions are based on the same reasoning, the conclusions presented at the end of this section hold for both graph types.

To vary intersection geometries, we randomize the two intersection positions and orientations on the fracture plane and we fix their size to $0.1,0.3$, and 0.5 normalized by the fracture size. For each intersection size, a thousand realizations are performed to obtain a large dataset covering various distances between intersections centers. The realizations where the two intersections cross on the fracture plane are rejected during generation because different heads cannot be imposed on the intersections if they share a common point, as previously discussed. A real estimation of the equivalent conductance is computed from the numerical simulations as $C_{\text {num }}=\frac{Q}{\Delta H}$, where $Q\left(\mathrm{~m}^{3} \mathrm{~s}^{-1}\right)$ is the flow and $\Delta H$ the head difference between intersections $(\mathrm{m})$, and is compared to the analytical conductance, $C_{j k}^{i}$, defined in Sec. III A 3 (Fig. 7, solid lines).

In addition, we compare $C_{\text {num }}$ to the lower-order conductance definition used in Karra et al. [32] (Fig. 7, dashed lines), which, unlike our definition depending on fracture size, relies on the intersection geometry only:

$$
C_{j k}^{i}=T_{i} \frac{l_{i j}+l_{i k}}{2\left\|\bar{X}_{i j}-\bar{X}_{i k}\right\|},
$$

with $T_{i}$ the fracture transmissivity $\left(\mathrm{m}^{2} \mathrm{~s}^{-1}\right)$, and $l_{i j}, \bar{X}_{i j}$ the size and center of the intersection $f_{i} \cap f_{j} \neq \emptyset$.

The benefit of the conductance definitions developed in Sec. III A 3 over the classic definition of Eq. (21) is demonstrated in Fig. 7. For all the intersection sizes, the presented conductance definition of Sec. III A 3 performs well (solid lines), with, however, a slight underestimation, that we

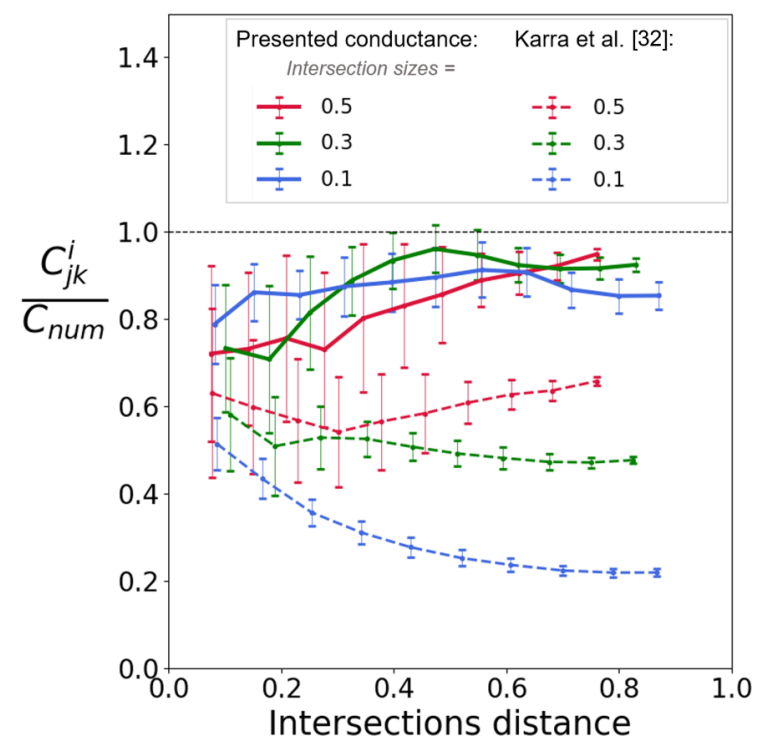

FIG. 7. Ratio between analytical and numerically solved conductances between two intersections on the same fracture plane as a function of the distance between intersection centers. Intersection sizes both equal $0.1,0.3$, and 0.5 normalized by the fracture size. The comparison is presented for the conductance developed in Sec. III A 3 (solid lines) and for the conductance used by Karra et al. [32] (dashed lines).

attribute to the geometrical simplification of the flowing area proposed in Sec. III A 1 (Fig. 3). Larger underestimation and more variability are observed when intersections get closer, possibly due to the angle between the intersections. When the two intersections are close and not parallel, high-pressure gradients may concentrate on some of their tips, creating a higher conductance than in the analytical solution. It is worth noting that the benefit of these developments over the lower order definition used in Karra et al. [32] is larger for smaller intersections. We can observe this by comparing, for example, the results for sizes equal to 0.1 times the fracture diameter (blues lines), for which the classic definition is underestimated by a factor $\sim 5$.

These observations are important for natural fracture networks, which are characterized by power-law distributions of fracture sizes [8]. Regarding the intersection sizes, we can note a lack of information since they are not measurable in the field. However, in DFN models, they usually also range over several orders $[39,40]$. In some models, they can also be very small compared to the fracture sizes, like in the universal fracture model (UFM, [19,36]) in which fractures grow and stop on other fractures, creating small intersections.

\section{ABILITY OF GRAPHS TO ESTIMATE FLOW IN LARGE DFNS}

In this section, we evaluate the ability of graphs using the conductance defined in Sec. III to estimate flow in a large DFN. We perform comparisons at both the DFN scale (permeability) and at the fracture scale (total flow exchanged by fracture, Sec. II B 2). In this study, all DFNs have a constant fracture transmissivity, equal to $1 \mathrm{~m}^{2} \mathrm{~s}^{-1}$. 
TABLE I. DFN generation parameters.

\begin{tabular}{|c|c|c|c|}
\hline & Poisson, constant size & Poisson, power-law size distribution & UFM \\
\hline Cubic system size & 50 & 50 & 50 \\
\hline Fracture size, $l(\mathrm{~m})$ & 3 & {$[1,50]$} & \\
\hline Powerlaw size distribution exponent, $a$ & & 4 & \\
\hline Target percolation parameter, $p(-)$ & {$[3,4,5,6,7]$} & {$[3,4,5,6,7]$} & \\
\hline Initial nuclei size $(\mathrm{m})$ & & & 1 \\
\hline Fracture growth stops on system borders & & & yes \\
\hline Nucleation rate, $\dot{n}\left(\mathrm{~s}^{-1}\right)$ & & & 0.01 \\
\hline Growth exponent, $e$ & & & 3 \\
\hline Growth rate parameter, $C$ & & & 1 \\
\hline Target growth time $t$ (s) & & & {$[0.5,1,3,7,10]$} \\
\hline Total number of realizations & 20 for each percolation & 20 for each percolation & 5 for each time \\
\hline Number of unconnected realizations & 0 & 8 for $p=3$ & 1 for $t=0.5$ \\
\hline
\end{tabular}

In Sec. III, we have defined the conductance for the ideal case of fractures in series, i.e., fractures have two intersections with other fractures and carry a unique flowing path. In reality, DFNs are characterized by a complex connectivity. Therefore, DFN fractures can have more than two intersections and carry many flowing paths, which could induce biases in the graph flow estimations. To evaluate these biases, we apply the methodology defined in Sec. II to different DFN types, that are characterized by different topologies. Later in this section, we increase the network topological complexity by looking first at stochastic DFNs with constant fracture sizes (Sec. IV A), second at stochastic DFNs with a power-law size distribution (Sec. IV B), and finally at genetic models, called UFMs, which are power-law size distributed networks with a specific spatial organization of the fractures (Sec. IV C).

The stochastic DFNs are generated with a targeted density, i.e., fractures are placed one by one into the system until the desired density value is obtained. In this study, we use the percolation parameter, $p$, as a dimensionless measure of density, instead of the classic measure of total fracture surface per unit volume $\left(P_{32}\left(\mathrm{~m}^{-1}\right)\right.$ [41]). The dimensionless density is defined as [42-44]

$$
p=\frac{1}{V} \frac{\pi^{2}}{8} \sum_{i} l_{i}^{3},
$$

where $V$ is the system volume and $l_{i}$ the fracture size. The percolation parameter is a good indicator of DFNs connectivity, regardless of the fracture size distribution [44]. Networks are connected when the percolation parameter is larger than the percolation threshold, estimated to be 2.5 by de Dreuzy et al. [42]. For the stochastic DFNs (Secs. IV A and IV B), we create twenty realizations of each network at the percolation parameter values 3, 4, 5, 6, and 7 (Table I).

The UFM networks (Sec. IV C) are generated with fracture nucleation, growth, and arrest protocol described in $[19,35,36]$. Their properties will depend on the nucleation rate relative to the fracture growth rate and on the growth time. We vary the targeted growth time to have UFM with a certain range of percolation parameters (Table I). More information about the generated UFM networks is provided in Sec. IV C.

Before performing the complete and graph flow simulations, DFN backbones are identified and isolated. We defined the backbone as the part of the network that carries flow. To this end, we first remove all clusters of fractures that are not connected to any constant head boundaries, and, second, we remove all the network dead ends by iteratively removing fractures having only one intersection with another fracture.

In Table II, we present some characteristics of the simulated DFNs. In addition to the DFN percolation, we also measure some topological indicators to characterize the DFN connectivity, like the average fracture degree, $\bar{n}$, and the maximum fracture degree, $n_{\max }$. The fracture degree is equal to the number of intersections on the fracture, calculated here after selecting the DFN backbone. The complete fracture degree distributions are also presented in the Appendix.

\section{A. Poissonian DFNs with constant fracture size}

The DFNs tested in this section have constant fracture sizes (Table I). They are characterized by a "quasi" regular structure, with fracture degrees being overall low [Appendix, Fig. 16(a)].

We represent the averaged ratio of the permeabilities estimated from the graph $\left(K_{G}\right)$ simulations and the complete simulations $\left(K_{\mathrm{DFN}}\right)$ as a function of the DFN percolation parameter (Fig. 8). The results show that almost no biases are observed in the graph predictions relative to the complete simulations. The fracture graph and the intersection graph present similar results (respectively blue squares and blue triangles).

For the intersection graph, we present the permeability results with and without the "multiple-paths" correction of the edge conductance defined in Sec. III A 3 (respectively full and empty triangles in Fig. 8). Without the correction, the graph flow predictions overestimate the complete simulation values. The magnitude of the overestimation increases with the percolation parameter. However, the graphs with the "multiple-paths" correction are in better agreement with the complete simulations, regardless of the percolation parameter.

We also compare the fracture total exchanged flow, $Q_{f}$ (Sec. II B 2), estimated by the complete and graph flow simulations (Fig. 9). Some very small values of $Q_{f}$ are measured in the complete and graph simulations (Fig. 9(a), $\left.\left[10^{-17}, 10^{-15}\right]\right)$, which are below machine precision and thus numerically zero. In the complete simulations, these values correspond to nonflowing fractures that remain in the DFN 
TABLE II. Statistics of geometrical and topological metrics of the simulated DFNs. Averages and standard deviations of the different realizations are presented for the two extreme targeted parameters: $p=3$ and 7 for the stochastic DFNs, and $t=0.5$ and $t=10$ for the UFM.

\begin{tabular}{|c|c|c|c|c|c|c|}
\hline & \multicolumn{2}{|c|}{ Poisson, constant size } & \multicolumn{2}{|c|}{$\begin{array}{l}\text { Poisson, power-law } \\
\text { size distribution }\end{array}$} & \multicolumn{2}{|c|}{ UFM } \\
\hline & $p=3$ & $p=7$ & $p=3$ & $p=7$ & $t=0.5$ & $t=10$ \\
\hline Measured percolation, $p$ & $3.1 \pm 0.0$ & $7.1 \pm 0.0$ & $3.1 \pm 0.1$ & $7.3 \pm 0.1$ & $5.0 \pm 0.5$ & $15.4 \pm 0.8$ \\
\hline Total fracture number & $11761 \pm 10$ & $27448 \pm 21$ & $33068 \pm 1910$ & $73070 \pm 5842$ & $691 \pm 17$ & $8381 \pm 74$ \\
\hline Fracture number in backbone & $8042 \pm 99$ & $27103 \pm 25$ & $2214 \pm 750$ & $42946 \pm 6819$ & $49 \pm 6$ & $5661 \pm 96$ \\
\hline Average fracture degree in backbone, $\bar{n}$ & $3.7 \pm 0.0$ & $7.1 \pm 0.0$ & $3.5 \pm 0.1$ & $4.2 \pm 0.1$ & $3.5 \pm 0.1$ & $4.7 \pm 0.1$ \\
\hline Max fracture degree in backbone, $n_{\max }$ & $12 \pm 1$ & $21 \pm 1$ & $110 \pm 34$ & $494 \pm 132$ & $12 \pm 1$ & $310 \pm 76$ \\
\hline
\end{tabular}

after the backbone selection, e.g., dead ends finishing with a loop. In the graph simulations, they can correspond to the same structures. However, the graphs predict zero flow in some fractures that do contain flow in the complete simulation [Fig. 9(a)]. We think that the flow on these fractures enters and exit through the same intersection, but the fractures are not part of a structural path. Therefore, the graphs predict zero flows.

We select the fractures that contain significant flow in both the complete and graph simulations to estimate the biases as a function of the fracture flow, $Q_{f, \text { DFN }}$ [Fig. 9(b)]. As we wish to focus on the structure of the DFN flows rather than on their magnitudes, the $Q_{f \text {,DFN }}$ are normalized by the complete simulation total flow [marked by $*$ in Fig. 9(b)]. Over $\sim 2$ orders of magnitude, $Q_{f, \text { DFN }}$ is well estimated by the graph flow simulation, and the variability remains low. But, for lower values of $Q_{f \text {,DFN }}$, the graphs overestimates on average the results, and the variability gets stronger.

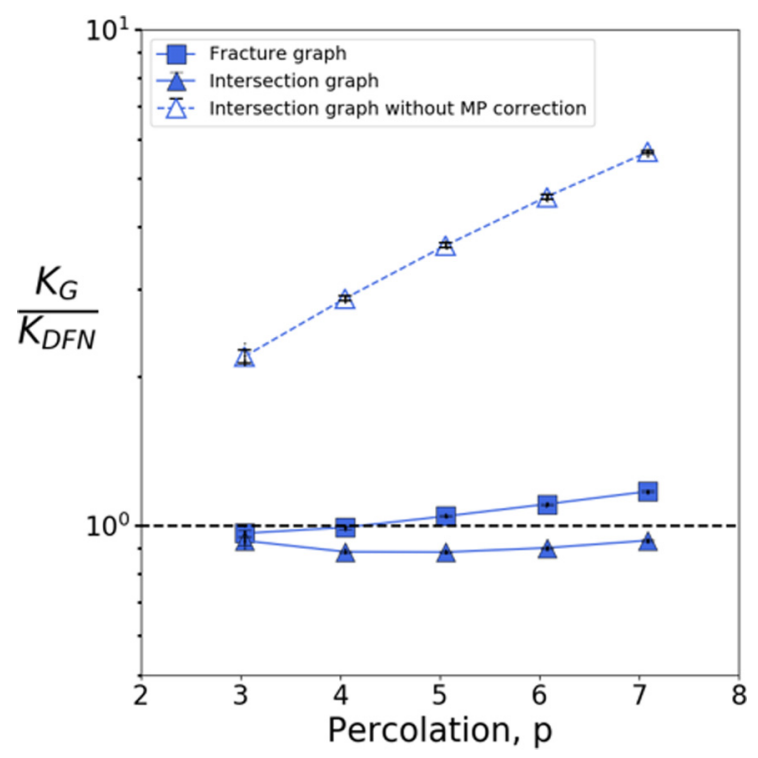

FIG. 8. Averaged ratio between graph and complete simulation permeabilities (resp. $K_{G}$ and $K_{\mathrm{DFN}}$ ), as a function of network percolation. The DFNs have constant fracture sizes. Twenty DFN realizations are performed for each percolation value $(3,4,5,6,7)$. The results of the fracture graph are presented with squares, and for the intersection graph with triangles. For the intersection graph, the results without the "multiple path" correction (Sec. III A 3) are also represented with empty triangles.

\section{B. Poissonian DFNs with power-law size distribution}

The DFNs in this section are characterized by a power-law density distribution of fracture sizes,

$$
n(l)=\alpha l^{-a} .
$$

$n(l) d l$ is the number of fractures per unit volume, whose size is in the range $[l, l+d l], a$ is the exponent, fixed to 4 , and $\alpha$ is the density term. Such DFNs are also characterized by a power-law distribution of the fracture degree [Appendix, Fig. 16(b)], which indicates that a few fractures have a very high connection number [45].

In this complex structure, the permeability from the graph simulation $\left(K_{G}\right)$ is slightly larger than the permeability from the complete simulation $\left(K_{\mathrm{DFN}}\right)$, and the difference increases with the DFN percolation parameter (Fig. 10). In the fracture graph, the overestimation (quantified by the ratio $\frac{K_{G}}{K_{\mathrm{DFN}}}$ ) ranges on average from $\sim 1.5$ for the lowest percolation to $\sim 2.1$ for the highest percolation (Fig. 10, yellow squares). It is slightly smaller in the intersection graph, from $\sim 1.2$ to $\sim 1.8$ (Fig. 10, yellow triangles) after applying the "multiple-paths" correction (Sec. III A 3).

By comparing the fracture total exchanged flow, $Q_{f}$, we observe that there are a large number of fractures that do not have flow within them in the fracture graph [Fig. 11(a), red dots] but are flowing in the complete simulation. This difference is not observed in the intersection graph (green dots). Therefore, this bias results from the fracture graph architecture. One can explain this by considering the alternative flowing paths formed by small fractures around a unique large fracture. The structures formed by these fractures are not necessarily dead ends. They can also be a loop structure with their flowing entrance and exit located on a single large fracture. In this case, the edges formed by fractures nodes create structures that cannot flow because they are linked to the same fracture node. This effect is more pronounced in the DFNs with power-law size distribution because a lot of flowing structures tend to be connected to a unique large fracture. These differences and discrepancies highlight an important limitation of using the fracture graph to resolve fracture network flow.

Next, we isolate fractures that have flow both in the complete and graph simulations, and we plot the graph biases $\left(\frac{Q_{f, G}}{Q_{f D F N}}\right)$ as a function of the real fracture flow, $Q_{f, \mathrm{DFN}}$ [Fig. 11(b)]. The plots present the same trends as in the constant sized networks, i.e., good predictions are obtained for larger flows $\left(Q_{f, \mathrm{DFN}}^{*}>10^{-3} ; *\right.$ means normalized by the 
(a)

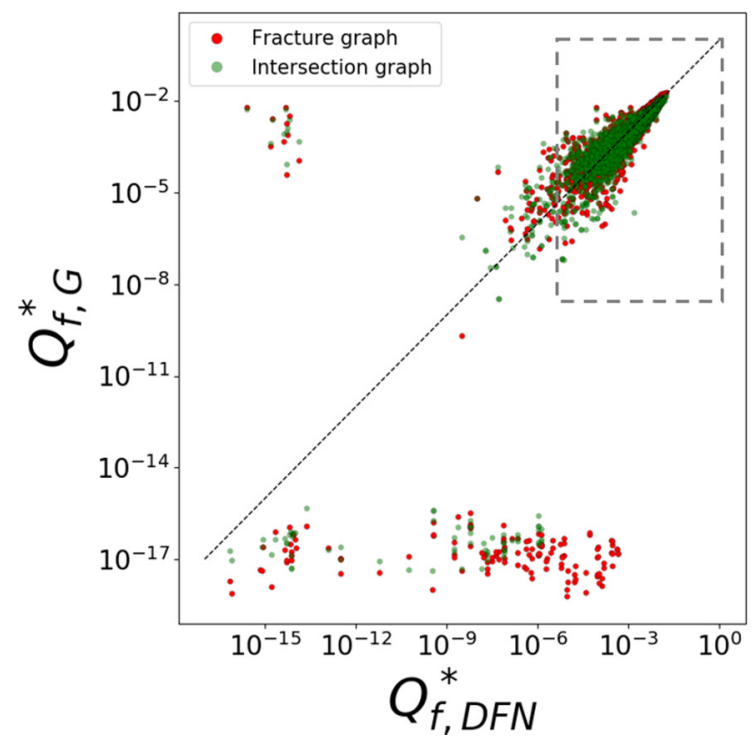

(b)

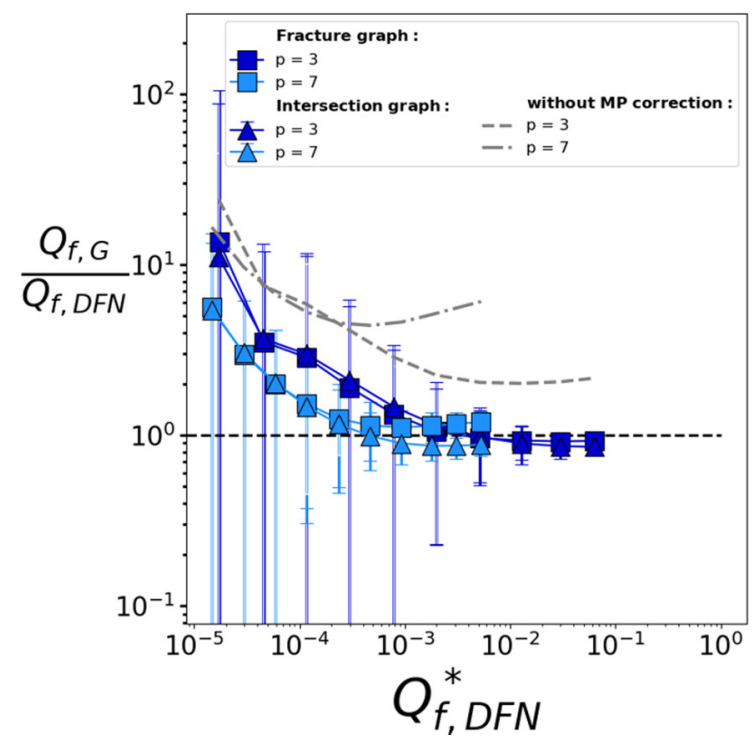

FIG. 9. Fracture total exchanged flow, $Q_{f}\left(\mathrm{~m}^{3} \mathrm{~s}^{-1}\right)$, in DFNs with constant fracture sizes. (a) Comparison between graph $\left(Q_{f, G}\right)$ and complete simulations $\left(Q_{f, \mathrm{DFN}}\right)$ in one DFN realization with percolation 5. (b) Fracture with major flows are selected [dashed square in (a)] and the averaged ratio between $Q_{f, G}$ and $Q_{f, \mathrm{DFN}}$ is represented as a function of $Q_{f, \mathrm{DFN}}$. Twenty DFN realizations are performed for each percolation value $(3,7)$. The results of the fracture graph are presented with squares, and for the intersection graph with triangles. The results are normalized by the total flow from the complete simulation (marked by *).

DFN total flow) and overestimations for small flow $\left(Q_{f, \text { DFN }}^{*}<\right.$ $\left.10^{-3}\right)$.

In the intersection graph, the fracture flow distribution is improved by the "multiple-paths" correction defined in

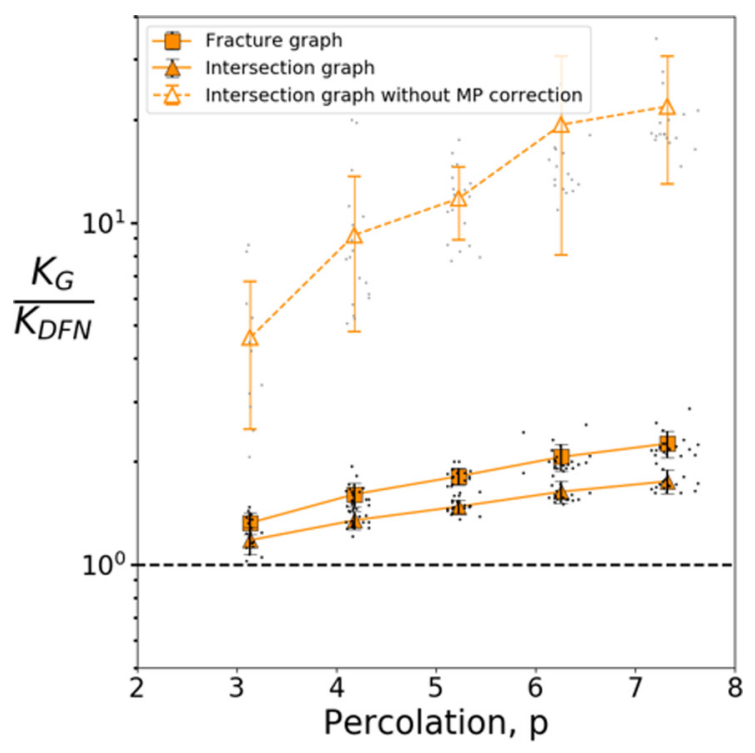

FIG. 10. Averaged ratio between graph and complete simulations permeability (resp. $K_{G}$ and $K_{\mathrm{DFN}}$ ), as a function of network percolation. The DFNs have a power-law size distribution with exponent 4. Twenty DFN realizations are performed for each percolation value $(3,4,5,6,7)$. The results of the fracture graph are presented with squares, and for the intersection graph with triangles. For the intersection graph, the results without the "multiple path" correction (Sec. III A 3) are also presented with empty triangles.
Sec. III A 3. Without this correction, major flows present stronger graph biases than smaller flows [gray dashed lines, Fig. 11(b)], thereby indicating that this correction is critical in the intersection graph, not only to estimate properties at a large scale, like DFN permeability, but also to estimate properties at a smaller scale, like the correct fracture flow distribution, $Q_{f}$.

The overestimations of the graph simulations relative to the complete simulations can be attributed to the fact that the edge conductances have been defined for a case of fracture with two intersections and do not consider that this surface may have to be shared between several flowing intersections. In this latter case, the edge conductance should overestimate the flow on the fracture plane. To check if a positive correlation exists between the intersection number on the fracture and the graph biases, we plot the ratio $\frac{Q_{f, G}}{Q_{f D F N}}$ as a function of the fracture degree, $n_{i}$ (Fig. 12). However, even if the fracture degree distribution is very wide in this DFN type, no positive correlation is observed: the highest overestimations are observed for the smallest degrees, similar to the comparison with fracture flow in Fig. 11(b). A possible explanation for this result is that, in one realization, major flow biases happening in fractures with high connectivity influence the rest of the fractures.

\section{UFM networks}

UFM is a genetic fracture network model, where fractures are generated with nucleation, growth, and arrest processes $[19,36]$. The nucleation consists of generating fracture nuclei with a Poissonian process for their position and orientations, at a given rate $\dot{n}\left(\mathrm{~s}^{-1}\right)$. Then, fracture nuclei grow with at a rate $v\left(\mathrm{~m} \mathrm{~s}^{-1}\right)$ that is function of the fracture size $l: v(l)=$ $C l^{e}$, where $e$ is the growth exponent and $C$ the growth rate 
(a)

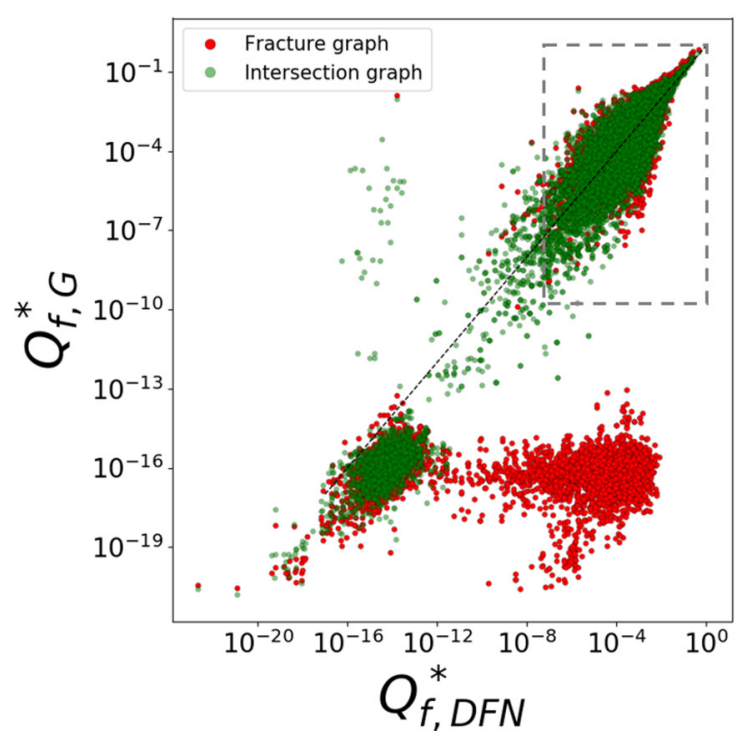

(b)

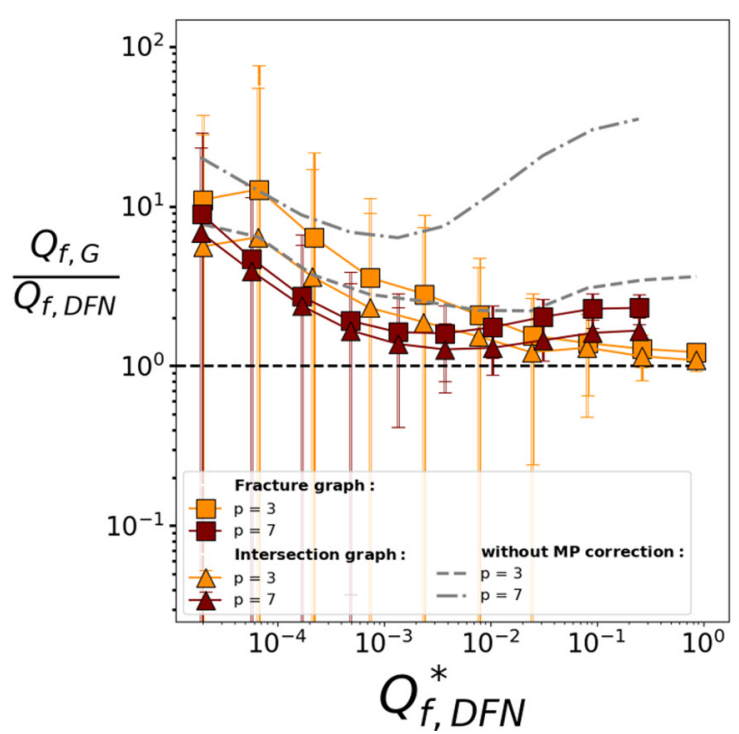

FIG. 11. Fracture total exchanged flow, $Q_{f}\left(\mathrm{~m}^{3} \mathrm{~s}^{-1}\right)$, in DFNs with powerlaw fracture size distribution. (a) Comparison between graph $\left(Q_{f, G}\right)$ and complete simulations $\left(Q_{f, \mathrm{DFN}}\right)$ in one DFN realization with percolation 5. (b) Fracture with major flows are selected (dashed square on Figure a)) and the averaged ratio between $Q_{f, G}$ and $Q_{f, \mathrm{DFN}}$ is represented as a function of $Q_{f, \mathrm{DFN}}$. Twenty DFN realizations are performed for each percolation value $(3,7)$. The results of the fracture graph are presented with squares, and for the intersection graph with triangles. The results are normalized by the total flow from the complete simulation (marked by *).

parameter. Fractures stop growing if they cross another fracture with a larger size. In this study, $\dot{n}$ is chosen very small relative to $C$ (Table I) so that fractures grow and stop one after

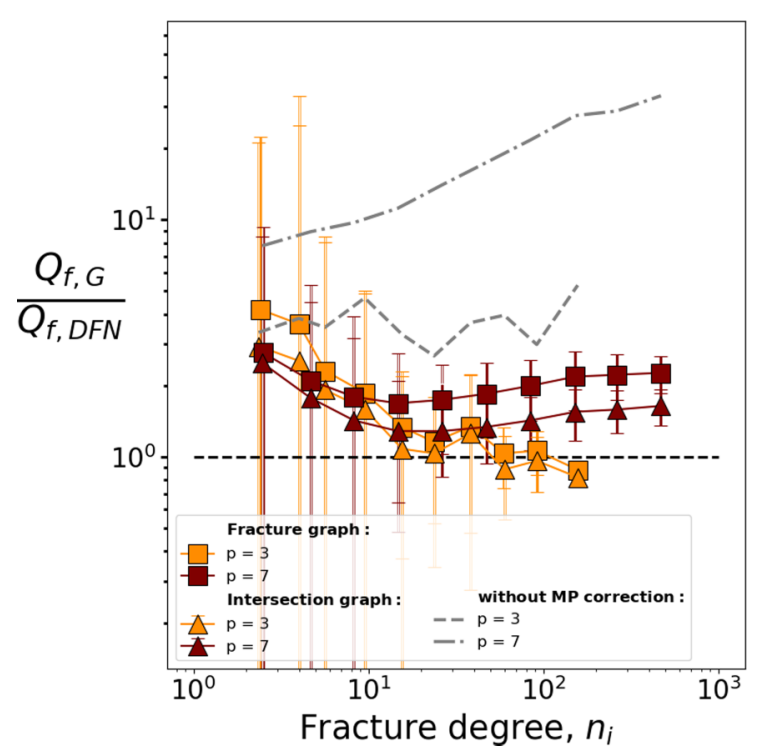

FIG. 12. Fracture total exchanged flow, $Q_{f}\left(\mathrm{~m}^{3} \mathrm{~s}^{-1}\right)$, in DFNs with power-law fracture size distribution. Fractures with major flows are selected [dashed square in Fig. 11(a)] and the averaged ratio between $Q_{f, G}$ and $Q_{f, \text { DFN }}$ is represented as a function of the fracture degree. Twenty DFN realizations are performed for each percolation value $(3,7)$. The results of the fracture graph are presented with squares, and for the intersection graph with triangles. The results are normalized by the total flow from the complete simulation (marked by *). the other. The process leads to fractures with a power-law size distribution with an exponent of -4 , and a large number of $\mathrm{T}$ intersections (one fracture abutting another) [35]. Regarding the topology, the degree distribution is also power-law shaped (Appendix, Fig. 16), but fractures present a lower degree (fewer intersections) than in an equivalent Poissonian network [35]. The UFM is also characterized by numerous intersections whose size is much smaller than the fracture size because of the arrest rule. We take UFM networks as examples of complex structures with characteristics different from the Poissonian networks described in the previous section.

As for the Poissonian network with power-law size distribution, permeabilities from the graph simulations $\left(K_{G}\right)$ are overestimated relative to the complete simulation $\left(K_{\mathrm{DFN}}\right)$, and the overestimation increases with the DFN percolation parameter [Fig. 13(a)]. However, the comparison with the other DFN types (Secs. IV A and IV B) indicates that the percolation parameter does not explain the overestimation because the evolution of $\frac{K_{G}}{K_{\mathrm{DFN}}}$ with this parameter is different within each DFN type.

As stated earlier in Sec. IV B, the conductance defined for a fracture case with two intersections hypothetically overestimates the complete flow simulation in DFNs having fractures with many flowing intersections. However, there is no correlation between the graph flow biases measured at fracture scale with the intersection number of the fracture, or fracture degree (Fig. 12), because the flow is mostly influenced by the best connected fractures present in the DFN. A better way to obtain a correlation between the graph biases and the fracture degree is to observe the effect of these most connected fractures on the graph biases. To this end, we represent $\frac{K_{G}}{K_{\mathrm{DFN}}}$ as a function of the maximum fracture degree present in the DFN, $n_{\max }$, for the three DFN types [Fig. 13(b)]. The 
(a)

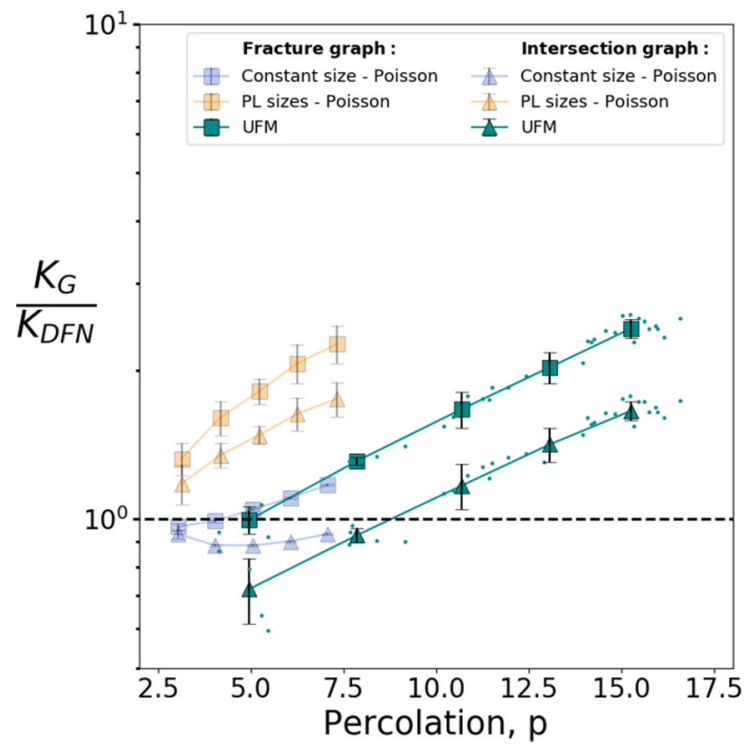

(b)

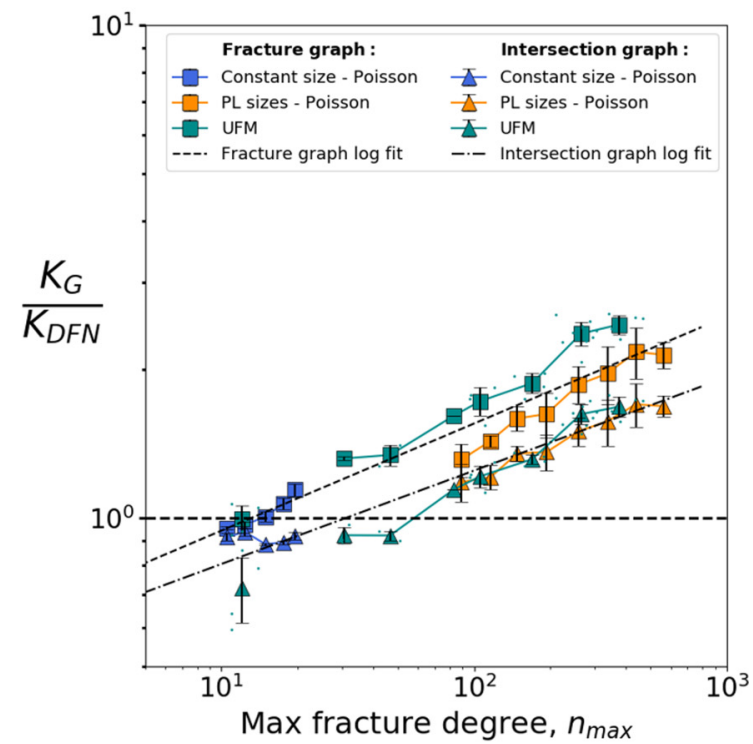

FIG. 13. Averaged ratio between graph and complete simulations permeability (resp. $K_{G}$ and $K_{\mathrm{DFN}}$ ), as a function of network percolation (a), and as a function of the network maximum fracture degree (b). The DFNs are UFM networks (cyan). The results of the fracture graph are presented with squares, and for the intersection graph with triangles.

results indicate a positive correlation between $\frac{K_{G}}{K_{\mathrm{DFN}}}$ and $n_{\max }$, which is common to the three DFN types. This confirms that the conductance defined in Sec. III overestimates the flow when fractures have many intersections. However, this result also indicates that the overestimation is rather slight: it reaches only values of $\sim 2.2$ and $\sim 1.8$ respectively for the fracture graph and the intersection graph, for fractures that are highly connected $\left(n_{i} \sim 400\right)$. The method can thus still predict correct flow orders, even when the DFNs have some highly connected fractures. Besides, the correlation can be quantified by a power-law relationship with exponents $\sim 0.22$ for the fracture graph and $\sim 0.19$ for the intersection graph (gray dashed lines in [Fig. 13(b)]), which could be used as an additional correction factor based on the highest fracture degree measured in the DFN.

Similar to the Poissonian power-law network (Sec. IV B), a comparison of the total flow exchanged by fracture indicates that some zero-flow artifacts are observed for the (a)

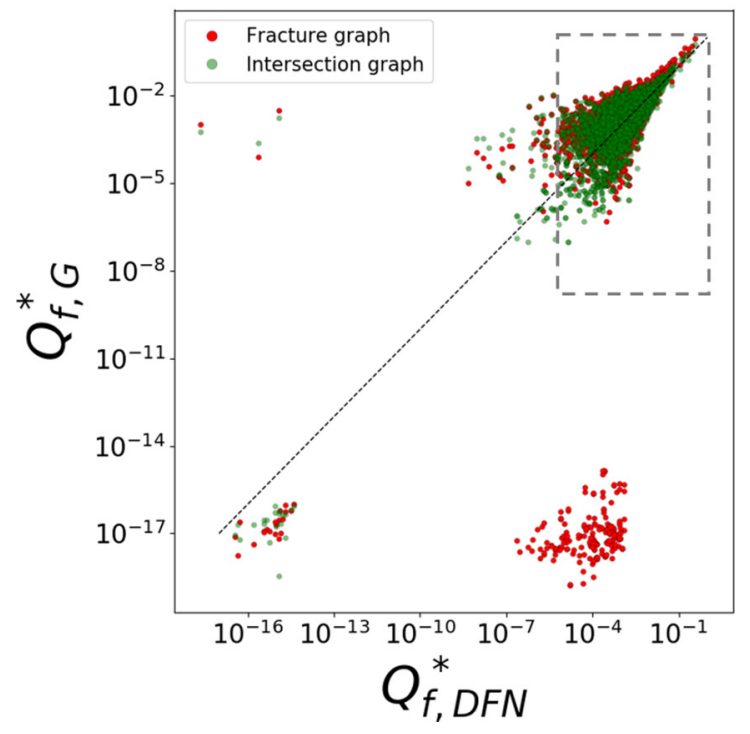

(b)

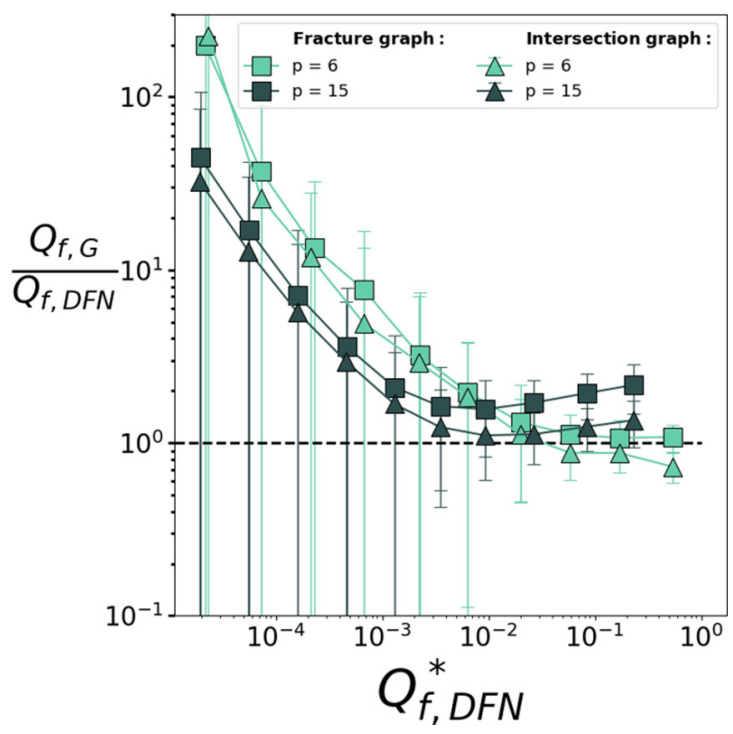

FIG. 14. Fracture total exchanged flow, $Q_{f}\left(\mathrm{~m}^{3} \mathrm{~s}^{-1}\right)$, in UFM networks. (a) Comparison between graph $\left(Q_{f, G}\right)$ and complete simulations $\left(Q_{f, \mathrm{DFN}}\right)$ in one UFM realization. (b) Fracture with major flows are selected [dashed square in (a)] and the averaged ratio between $Q_{f, G}$ and $Q_{f, \mathrm{DFN}}$ is represented as a function of $Q_{f, \mathrm{DFN}}$. The results of the fracture graph are presented with squares, and for the intersection graph with triangles. The results are normalized by the total flow from the complete simulation (marked by *). 
fracture graph [red dots, Fig. 14(a)]. After selecting the flowing fractures [Fig. 14(b)], the graph estimations are close to the complete simulation values over 2 orders of magnitude. However, as in Poissonian DFNs, both graphs overestimate the results for lower values of $Q_{f \text {,DFN }}$.

\section{CONCLUSION}

We have developed an analytical definition of the edge conductance for use in resolving flow in graphs that are equivalent to three-dimensional DFNs, in the case where the transmissivity by fractures is constant. Two graph types were analyzed in this study: a graph of fractures and a graph of intersections. In both graph types, we have derived an edge conductance equivalent to the fracture flowing surface by including the fracture size in the definition. We have observed, first at a single fracture scale, that this definition of the conductance is adapted to predict the flow through one fracture with two intersections across a wide range of intersection configurations. Besides, this definition is more accurate than previous definitions [32], mostly when the intersections are small compared to the fracture size.

The fracture graph and the intersection graph are two possible simplified representations of a DFN; however, the way they are constructed causes different issues when solving flow directly with the graph. The intersection graph creates an $n$-clique on each fracture having $n$ intersections (a clique is characterized by every two nodes being connected). This feature implies that, as soon as the number of intersections is higher than 2 on a fracture, many graph paths exist from one intersection node to another on this fracture. In turn, the equivalent flowing surface artificially increases and produces strong biases in the graph estimations. Therefore, in the intersection graph, the initial conductance definition is completed by including a correction factor that accounts for the number of intersections on a fracture ("multiple-paths correction"). This correction markedly improves the intersection graph flow estimations in every DFN type that were tested in this study. In the fracture graph, no such multiple paths exists and a correction of this type is therefore not needed. However, a disadvantage of using the fracture graph is that particular regions of the DFN are no-flow when they should not be. We have observed that this graph type does not permit solving the flow in parts of the DFN that are connected to a large unique fracture, which is often the case in DFNs with power-law fracture size distribution. In turn, the local flow exchange rates are incorrect in these regions.

The robustness of the conductance definitions was tested in a suite of large networks, by comparing both graph flow simulations to the complete DFN flow simulation. DFNs exhibit a range of connectivity complexity, which, in turn, can induce graph flow overestimations because fracture surfaces have to be partitioned into several flowing paths. We have evaluated these potential graph biases for DFNs with increasing connectivity complexity: stochastic DFNs with constant fracture sizes, stochastic DFNs with a power-law size distribution, and UFM networks. Two scales were evaluated: the DFN scale and the fracture scale. At the DFN scale, the topology, i.e., how fractures relate to each other, explains the measured flow biases in both graph types. A common positive correlation between the graph biases and the DFN maximum fracture degree was observed and quantified. However, these biases are relatively small in both graph types $(\times \sim[1,2.5])$ compared to the range of equivalent permeabilities that have been computed (on average $K \in\left[10^{-3}, 10^{-1}\right] \mathrm{m} \mathrm{s}^{-1}$ ), which makes the method promising for a quick estimate of large DFN equivalent permeabilities. At the fracture scale, no correlation could be highlighted between the fracture degree and graph flow biases.

We have also evaluated the ability of the graph to reproduce the DFN flowing structure by comparing graph flow and DFN flow at the fracture scale. In all the DFNs, the flow structure was respected in the graph over $\sim 2$ magnitude orders. However, for fractures carrying smaller flows $\left(Q_{f}^{*} \lesssim 10^{-2}-10^{-3}\right)$, the variability inevitably increases and the graphs on average overestimate the flow results.

An advantage of the graph is the reduced computational time required for the graph simulations. For example, the Poissonian DFNs with power-law size distribution and percolation 7 (Sec. IV B) has on average 43000 fractures. In the complete flow simulations, each network is meshed with about $5 \times 10^{6}$ cells, and it takes around $500 s$ to mesh and solve the flow. In the equivalent graphs, the complexity is reduced to average numbers of 44000 vertices in the fracture graph and 97000 in the intersection graph. The CPU times to generate one graph from the DFN and solve the flow are reduced to $3 s$ for the fracture graph, and $40 \mathrm{~s}$ for the intersection graph, which translates to speed-ups of $10^{2}$ and 10 times respectively.

If the flow is adequately estimated by the graph-based models, they can be used to reduce the cost of DFN modeling with little loss of information, thus making possible the calculation of flow in DFN models with fracture sizes spanning multiple orders of magnitude. The practical applications with graph-based models are to simplify the DFN structure before calculating flow or transport properties (e.g., selecting the network backbone or important paths), or to consider very large ensembles of DFN models with both high- and low-fidelity descriptions to quantify the variability and uncertainty in these systems (O'Malley et al. [33] and Berrone et al. [34]).

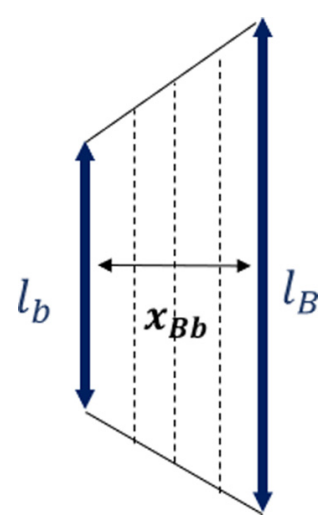

FIG. 15. Schema and notations used for the analytical definition of flow conductance between two parallel lines. 


\section{ACKNOWLEDGMENTS}

This work was funded by the CIFRE grant ITASCA Consultants SAS/ANRT (Association Nationale Recherche Technologie, France) 2017/1158, and partially supported by the Swedish Nuclear Fuel and Waste Management Co. (SKB). We thank the Fractory team (Rennes, France), particularly Romain Le Goc, Benoit Pinier, and Etienne Lavoine, for the developments in the DFN.Lab software that were needed for this study. We thank Jan-Olof Selroos from SKB for his support and the interesting discussions. We thank the Los Alamos National Laboratory for hosting one of our authors, D.D. which enabled collaborative work on this project. Constructive reviews from two anonymous reviewers are greatly appreciated.

\section{APPENDIX}

\section{Analytical definition of conductance between two parallel lines (trapezoid)}

We set $x \in\left[0, x_{B b}\right]$. We set constant heads $(m), H_{B}$ at the base of size $l_{B}$, and $H_{b}$ at the base of size $l_{b}$, and make the hypothesis that equipotential lines between $l_{b}$ and $l_{B}$ are parallel (dashed lines in Fig. 15 )

The total flow $Q$ from $l_{b}$ to $l_{B}\left(\mathrm{~m}^{3} \mathrm{~s}^{-1}\right)$ can be written from Darcy's law as

$$
Q=T l(x) \frac{d h}{d x} .
$$

with $l(x)$, the width through which the fluid flows at location $x$, defined as

$$
l(x)=l_{b}+\frac{x}{x_{B b}}\left(l_{B}-l_{b}\right) .
$$

$T$ is the fracture transmissivity $\left(\mathrm{m}^{2} \mathrm{~s}^{-1}\right)$ and $\frac{d h}{d x}$ the head gradient along $x$.
We can write

$$
h_{B}-h_{b}=\int_{0}^{x_{B b}} d h .
$$

Combining (A1) and (A3) we obtain

$$
h_{B}-h_{b}=\frac{Q}{T} \int_{0}^{x_{B b}} \frac{d x}{l(x)} .
$$

The equivalent conductance $C\left(\mathrm{~m}^{2} \mathrm{~s}^{-1}\right)$ between both bases is defined as

$$
C=\frac{Q}{h_{B}-h_{b}} .
$$

Combining (A4), (A4), and (A5) we obtain

$$
\frac{1}{C}=\frac{1}{T} \int_{0}^{x_{B b}} \frac{d x}{l_{b}+\frac{x}{x_{B b}}\left(l_{B}-l_{b}\right)} .
$$

Solving the integral leads to the following expression:

$$
C=T \frac{l_{B}-l_{b}}{x_{B b}\left[\ln \left(l_{B}\right)-\ln \left(l_{b}\right)\right]} .
$$

$\ln (x)$ is the base $e$ logarithm.

\section{DFN fracture degree distributions}

For each DFN type simulated in the present study, we provide in Fig. 16 the average and standard deviation of the degree distributions computed in the different realisations. These representations highlight the different structures of the simulated DFNs. (a)

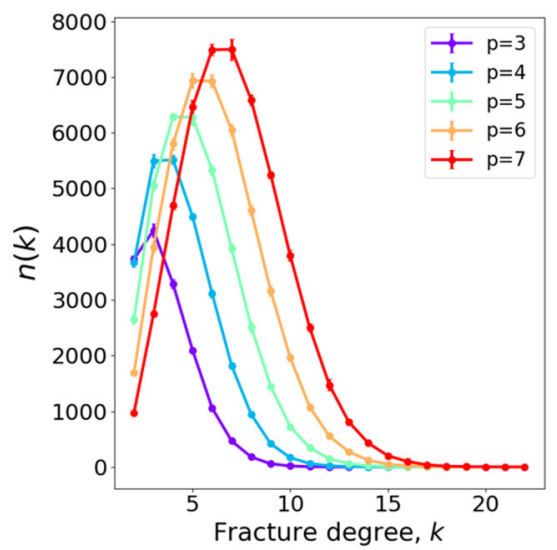

(b)

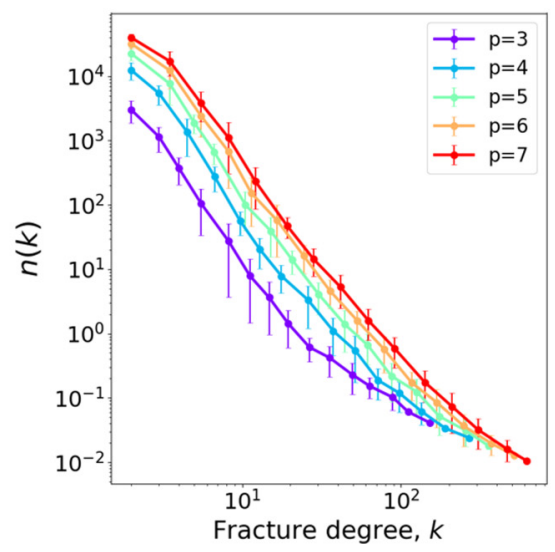

(c)

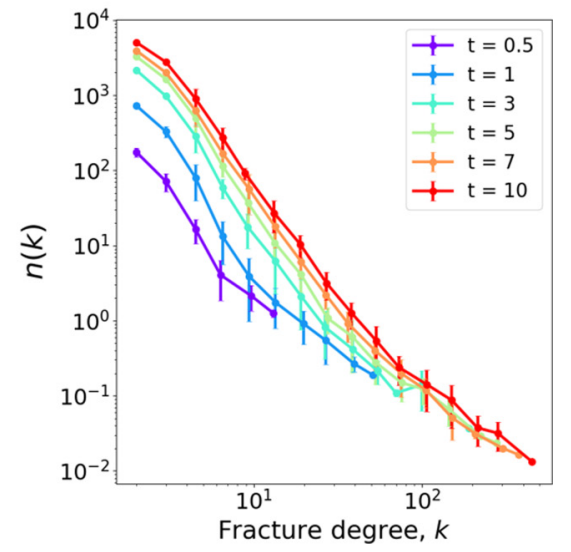

FIG. 16. Averaged fracture degree distribution in (a) Poissonian DFNs with constant fracture size, (b) Poissonian DFNs with power-law size distribution, and (c) UFM. The fracture degree is computed after backbone selection; isolated clusters and dead ends are removed from the network. Results are presented for different DFN percolations $p$, except for the UFM, in which results are presented for the different network growth times we have simulated. 
[1] R. Albert and A.-L. Barabási, Rev. Mod. Phys. 74, 47 (2002).

[2] A.-L. Barabási, R. Albert, and H. Jeong, Physica A 281, 69 (2000).

[3] M. E. J. Newman, Phys. Rev. E 64, 016131 (2001).

[4] H. Jeong, S. P. Mason, A.-L. Barabási, and Z. N. Oltvai, Nature (London) 411, 41 (2001).

[5] L. Valentini, D. Perugini, and G. Poli, Physica A 377, 323 (2007).

[6] C. A. Andresen, A. Hansen, R. Le Goc, P. Davy, and S. M. Hope, Front. Phys. 1, 7 (2013).

[7] S. M. Hope, P. Davy, J. Maillot, R. Le Goc, and A. Hansen, Front. Phys. 3, 75 (2015).

[8] E. Bonnet, O. Bour, N. E. Odling, P. Davy, I. Main, P. Cowie, and B. Berkowitz, Rev. Geophys. 39, 347 (2001).

[9] J. C. Long, J. Remer, C. Wilson, and P. Witherspoon, Water Resour. Res. 18, 645 (1982).

[10] R. A. Freeze, Water Resour. Res. 11, 725 (1975).

[11] D. L. Y. Wong, F. Doster, S. Geiger, and A. Kamp, Water Resour. Res. 56, e2019WR025774 (2020).

[12] P. Davy, O. Bour, J.-R. De Dreuzy, and C. Darcel, Geol. Soc. Spec. Publ. 261, 31 (2006).

[13] Hyman, M. Dentz, A. Hagberg, and P. K. Kang, J. Geophys. Res. Solid Earth 124, 1185 (2019).

[14] J.-O. Selroos and S. Follin, Hydrogeol. J. 22, 295 (2014).

[15] S. P. Neuman, Hydrogeol. J. 13, 124 (2005).

[16] T. Doe, R. McLaren, and W. Dershowitz, in Proceedings of the 39th Workshop on Geothermal Reservoir Engineering (Curran Associates, Inc., Stanford, 2014), p. 24.

[17] S. Karra, N. Makedonska, H. S. Viswanathan, S. L. Painter, and J. D. Hyman, Water Resour. Res. 51, 8646 (2015).

[18] J. D. Hyman et al., Phil. Trans. R. Soc. A 374, 20150426 (2016).

[19] P. Davy, R. Le Goc, C. Darcel, O. Bour, J.-R. De Dreuzy, and R. Munier, J. Geophys. Res. Solid Earth 115 (2010).

[20] Q. Lei, J.-P. Latham, and C.-F. Tsang, Comput. Geotech. 85, 151 (2017).

[21] M. C. Cacas, E. Ledoux, G. D. Marsily, A. Barbeau, P. Calmels, B. Gaillard, and R. Magritta, Water Resour. Res. 26, 491 (1990).

[22] M. C. Cacas, E. Ledoux, G. D. Marsily, B. Tillie, A. Barbeau, E. Durand, B. Feuga, and P. Peaudecerf, Water Resour. Res. 26, 479 (1990).

[23] W. Dershowitz and C. Fidelibus, Water Resour. Res. 35, 2685 (1999).

[24] A. W. Nordqvist, Y. Tsang, C. Tsang, B. Dverstorp, and J. Andersson, Water Resour. Res. 28, 1703 (1992).

[25] J. C. Long, P. Gilmour, and P. A. Witherspoon, Water Resour. Res. 21, 1105 (1985).
[26] H. S. Viswanathan, J. Hyman, S. Karra, D. O'Malley, S. Srinivasan, A. Hagberg, and G. Srinivasan, Water Resour. Res. 54, 6085 (2018).

[27] J. D. Hyman, A. Hagberg, D. Osthus, S. Srinivasan, H. Viswanathan, and G. Srinivasan, Multiscale Model. Simul. 16, 1948 (2018).

[28] J. D. Hyman, A. Hagberg, G. Srinivasan, J. MohdYusof, and H. Viswanathan, Phys. Rev. E 96, 013304 (2017).

[29] S. Srinivasan, J. Hyman, S. Karra, G. Srinivasan, and H. Viswanathan, in 2nd International Discrete Fracture Network Engineering Conference, June 2018, Seattle (American Rock Mechanics Association, Alexandria, VA, 2018).

[30] S. Srinivasan, S. Karra, J. Hyman, H. Viswanathan, and G. Srinivasan, Comput. Geosci. 23, 617 (2019).

[31] M. Valera, Z. Guo, P. Kelly, S. Matz, V. A. Cantu, A. G. Percus, J. D. Hyman, G. Srinivasan, and H. S. Viswanathan, Comput. Geosci. 22, 695 (2018).

[32] S. Karra, D. O'Malley, J. D. Hyman, H. S. Viswanathan, and G. Srinivasan, Phys. Rev. E 97, 033304 (2018).

[33] D. O’Malley, S. Karra, J. Hyman, H. S. Viswanathan, and G. Srinivasan, Water Resour. Res. 54, 3758 (2018).

[34] S. Berrone, J. Hyman, and S. Pieraccini, Water Resour. Res. 56, e2019WR026493 (2020).

[35] J. Maillot, P. Davy, R. Le Goc, C. Darcel, and J.-R. De Dreuzy, Water Resour. Res. 52, 8526 (2016).

[36] P. Davy, R. Le Goc, and C. Darcel, J. Geophys. Res. Solid Earth 118, 1393 (2013).

[37] W. Dershowitz and H. Einstein, Rock Mech. Rock Eng. 21, 21 (1988).

[38] B. Pinier, R. Le Goc, and P. Davy (unpublished).

[39] Y. F. Alghalandis, C. Xu, and P. A. Dowd, in Proceedings of the 2011 Australian Geothermal Energy Conference (Geoscience Australia, Canberra, 2011), p. 15.

[40] J. Zhan, P. Xu, J. Chen, W. Zhang, C. Niu, and X. Han, Int. J. Rock Mech. Min. Sci. 89, 116 (2016).

[41] W. S. Dershowitz and H. H. Herda, in The 33th US Symposium on Rock Mechanics (USRMS) (American Rock Mechanics Association, Alexandria, VA, 1992).

[42] J.-R. de Dreuzy, P. Davy, and O. Bour, Phys. Rev. E 62, 5948 (2000).

[43] O. Bour and P. Davy, Water Resour. Res. 34, 2611 (1998).

[44] O. Bour and P. Davy, Water Resour. Res. 33, 1567 (1997).

[45] J. D. Hyman, M. Dentz, A. Hagberg, and P. K. Kang, Phys. Rev. Lett. 123, 248501 (2019). 